International Journal of Recent advances in Physics (IJRAP) Vol.4, No.4, November 2015

\title{
Surface Polaritons in GaAs/AlGaAs/LH HeTrojunCTION STRUCTURE IN A High MAGNETIC FIELD
}

\author{
Majdi S. Hamada ${ }^{1}$, Ali. H. EL-Astal ${ }^{1}$ and Mohamed. M. Shabat ${ }^{2}$ \\ ${ }^{1}$ Department of Physics, Al-Aqsa University, Gaza, P.O. Box 4015, \\ Gaza Strip, Palestinian Authority \\ 2 Department of Physics, The Islamic University of Gaza, P.O. Box 108, Gaza Strip, \\ Palestinian Authority
}

\begin{abstract}
The surface polaritons (SP) variation in Ga As/ Al Ga As/ LH hetrojunction composition in the presence of a strong transverse quantized magnetic field is estimated using the quantum Hall effect case. The dispersion characteristics of the SPs are investigated using the dielectric constants values of the Ga As and the Alx Ga 1-x As media and the defined thickness, the Alx Ga 1-x As medium. The dispersion behaviours calculated results are listed for considered cases. It was shown that the frequency values against the wave vector values are affected in a strong manner by changing thickness, of the $A l_{x} G a_{1-x}$ - As media and by changing the variation of the dielectric constants of Ga As against the Alx Ga 1-x As. The significance effects of the use of the left-handed (LH) medium as an upper layer of the proposed composition was demonstrated; the frequency values are remarkably increased using LH material as an upper layer. It was noticed that at certain conditions of the LH upper layer composition, similar results have been obtained such as found by using dielectric upper layer.
\end{abstract}

\section{KEYWORDS}

Dispersion relation, Surface Polaritons, Left-Handed Material, Hetrojunction Structure

\section{INTRODUCTION}

According to rapid advance in crystal-growth procedures, such as molecular beam epitaxy and metal-organic chemical vapour deposition, the importance of the collective electromagnetic excitation in two-dimensional electron systems (2DES) has been strongly enhanced. This leads to the special interest in the surface polaritons (SP) which are non- irradiate electromagnetic waves existed at the 2DES [1-3].

When an external magnetic field B is applied, (in such a way which is perpendicular to the 2DES) leads to important characteristics. For instance, the phase velocity $v_{p h}=\omega / k$, and the group velocity, $v_{g}=\partial \omega / \partial k$, of the SPs decrease remarkably in the neighborhood of the cyclotron resonance (CR). $\omega$ and $\mathrm{k}$ are the frequency and the in-plane value of the wave vector of the SPs, respectively [4]. In this case, the SPs become slow waves. Of great importance are the characteristics of the SPs in high values of magnetic fields, under the conditions which produce the integer quantum Hall effect $[5,6]$. In this case, all of the values of the conductivity tensor of the 2DES are quantized, i.e. they exhibit stepwise behavior as the magnetic field varies. Due to this, the dispersion behaviors of the SPs are quantized too. Specially, when the magnetic field value is varied, the SP group velocity is demonstrated in a quantized variation in the 
neighborhood of the CR. The value of the steps is proportional to the fine-structure constant, $\alpha=e^{2} / \hbar c$, where $\mathrm{e}$ is the electron charge, and $\mathrm{c}$ is the velocity of light.

It is considered here that the dispersion characteristics of the SPs are studied in such a case which is assumed that a $2 \mathrm{DES}$ is positioned into an infinite homogeneous medium where dielectric constant is $\varepsilon$ [5,6]. In fact, the real picture is more complicated. A chosen doped $\mathrm{Al}_{\mathrm{x}} \mathrm{Ga}_{1-\mathrm{x}} \mathrm{As}$ medium has a finite thickness. This leads to the fact the electrons which form the 2DES are positioned between a GaAs medium (this layer forms the substrate, and its thickness can be considered to be infinite) and a $\mathrm{Al}_{\mathrm{x}} \mathrm{Ga}_{1-\mathrm{x}} \mathrm{As}$ medium. Furthermore, the dielectric constants of GaAs and $\mathrm{Al}_{x} \mathrm{Ga}_{1-\mathrm{x}} \mathrm{As}$ are not the same $[7,8]$. In $[7,8]$ the ratio of the dielectric constants of the $\mathrm{Al}_{\mathrm{x}} \mathrm{Ga}_{1-\mathrm{x}} \mathrm{As}$ and the GaAs was chosen to be 0.95 . These parameters select the behavior of the SPs in the GaAs/ $\mathrm{Al}_{\mathrm{x}} \mathrm{Ga}_{1-\mathrm{x}} \mathrm{As}$ structure.

In these days, negative refraction in left-handed materials (LHMs) encourage researchers for high concern, giving an excellent picture for research in both theoretical and practical fields. More than six decades ago, the theory of the propagation of electromagnetic waves in such media developed by Veselago [9]. Pendry et. al. [10,11] triggered the beginning of such important field, this encouraged construction of the earlier LHM structure. His research group conducted many valuable experiments, in the microwave radiation. This research team used a structure of splitring resonators (SRRs) of metal. This SRRs have certain useful properties in selected conditioned preferred in the world of electronics and industry where the working medium has outstanding amazed feature, i.e., negative permeability $\mu$ [10]. Thin wires of metal was constructed in a way similar to a quasi-metal where it has a negative permittivity $\varepsilon$ at microwave radiation $[11,12]$. Smith et. al. [13] constructed, a left-handed materials where both $\varepsilon$ and $\mu$ have negative values in preferable radiation condition. Although the fact that many researchers concentrated on the study and investigation of LHM. IThere still many things are not well known [14-18]. The researchers give the important beginning of the study of both the theory and practice side of the topic. Thus surface polariton of a LHM [19], highlighting the characteristics of LHM spheres [20] or cylinders [21], or the characteristics of radiation propagation in LHM [22] are studied in the theory side, and in the practice concern $[13,23]$.

In this paper, we investigate the surface polaritons in a 2DES where the quantum Hall effect is applied, and where also the finite thickness of the $\mathrm{Al}_{\mathrm{x}} \mathrm{Ga}_{1-\mathrm{x}} \mathrm{As}$ medium and taking into consideration the great difference of the dielectric constants of $\mathrm{GaAs}$ and $\mathrm{Al}_{\mathrm{x}} \mathrm{Ga}_{1-\mathrm{x}} \mathrm{As}$. Because of the interested characteristics of LHM [24-28], we introduce a LHM upper layer in the proposed structure and study the characteristics of surface polaritons in GaAs/ AlGaAs/ LHM hetrojunction in a high magnetic field structure as shown in figure 1. 


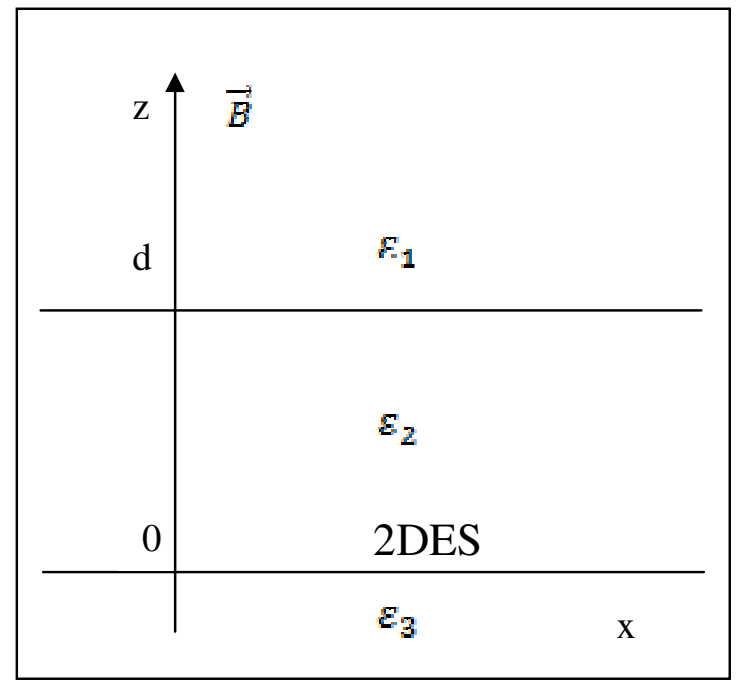

Fig.1: The geometry of the proposed structure. The semi-infinite medium 1 is the left-hand material layer with $\varepsilon_{1}$ and $\mu_{1}$; medium 2 is the $\mathrm{Al}_{\mathrm{x}} \mathrm{Ga}_{1-\mathrm{x}}$ As layer with the dielectric constant $\varepsilon_{2}$; the semi-infinite medium 3 is the GaAs layer with the dielectric

\section{THEORY: ELECTOMAGNETIC OF THE GaAs/ AlGaAs HETROJUNCTION IN A HIGH MAGNETIC FIELD}

Fig.1. shows our model structure, it consists of two semi-infinite media, with medium1 is LHM and dielectric constant $\varepsilon_{1}(\mathrm{z}>\mathrm{d})$ and medium $3(\mathrm{z}<0)$, with dielectric constant $\varepsilon_{3}$, separated by a thin layer (medium 2) with the thickness $d$ and the dielectric constant $\varepsilon_{2}$. Medium 2 is consists of $\mathrm{AlGaAs}$ semiconductor and medium 3 is also of GaAs semiconductor and these two mediums form the hetrojunction at the interface $\mathrm{z}=0$, i.e. 2DES is formed. The external quantized magnetic field, $\mathrm{B}$, is directed perpendicularly along $\mathrm{z}$-axis. The components of the electromagnetic fields are considered to be non-irradiative, so the propagation of the SPs in a 2DES in the media 1 and 3 were decreased exponentially as the distance $|z| \rightarrow \infty$.

To derive the dispersion equation which describe the SPs, two type of waves, TE and TM waves are taken into account, where the surface current is presented at the interface $\mathrm{z}=0$, which make TE and TM waves are mixed[29].

We assume that the electromagnetic waves are propagating along the $\mathrm{x}$-axis, and the components of TM waves in each media, 1,2,3, have

$$
\begin{gathered}
\mathrm{H}_{\mathrm{y}, 1}=\mathrm{H}_{1} \exp \left[i(k x-\omega t)-\mathrm{p}_{1}(\mathrm{z}-\mathrm{d})\right] \quad \mathrm{z}>\mathrm{d} \\
\mathrm{E}_{\mathrm{x}, 1}=\left(\mathrm{icp} / \omega \varepsilon_{1}\right) \mathrm{H}_{\mathrm{y}, 1} \quad \mathrm{z}>\mathrm{d} \\
\mathrm{E}_{\mathrm{z}, 1}=-\left(\mathrm{ck} / \omega \varepsilon_{1}\right) \mathrm{H}_{\mathrm{y}, 1} \quad \mathrm{z}>\mathrm{d}
\end{gathered}
$$


International Journal of Recent advances in Physics (IJRAP) Vol.4, No.4, November 2015

$$
\begin{gathered}
H_{y, 2}=\left[H_{2}^{(1)} \exp \left(p_{2} z\right)+H_{2}^{(2)} \exp \left(-p_{2} z\right)\right] \exp [i(k x-\omega t)] \quad 0<\mathrm{z}<\mathrm{d} \\
\mathrm{E}_{\mathrm{x}, 2}=-\left(\mathrm{ck} / \omega \varepsilon_{2}\right)\left(\partial H_{y, 2} / \partial z\right) \\
\mathrm{E}_{\mathrm{z}, 2}=-\left(\mathrm{ck} / \omega \varepsilon_{2}\right) \mathrm{H}_{\mathrm{y}, 2} \\
\mathrm{H}_{\mathrm{y}, 3}=\mathrm{H}_{3} \exp \left[\mathrm{i}(\mathrm{kx}-\omega \mathrm{t})+\mathrm{p}_{3} \mathrm{z}\right] \quad \mathrm{z}<0 \\
E_{x, 3}=-\left(i c p_{3} / \omega \varepsilon_{3}\right) H_{y, 3} \\
\mathrm{E}_{\mathrm{z}, 3}=\left(-\mathrm{ck} / \omega \varepsilon_{3}\right) \mathrm{H}_{\mathrm{y}, 3} \\
E_{y}=H_{x}=H_{z}=0 \quad p_{i}=\sqrt{k^{2}-\frac{\omega^{2}}{c^{2}} \varepsilon_{i} \mu_{i}} \quad i=1,2,3, \text { and } \mu_{2}=\mu_{3}=1 .
\end{gathered}
$$

In the case of non-radiative waves, the condition for the component of the wave vector $\mathrm{k}$ should hold:

$$
\operatorname{Re} p_{i} \succ 0 \quad i=1,3 \text {. }
$$

The components of the electromagnetic field for the non-radiative TE waves have the form

$$
\begin{gathered}
\mathrm{E}_{\mathrm{y}, 1}=\mathrm{E}_{1} \exp \left[\mathrm{i}(\mathrm{kx}-\omega \mathrm{t})-\mathrm{p}_{1}(\mathrm{z}-\mathrm{d})\right] \mathrm{z}>\mathrm{d} \\
\mathrm{H}_{\mathrm{x}, 1}=-\mathrm{I}\left(\mathrm{cp}_{1} / \omega \mu_{1}\right) \mathrm{E}_{\mathrm{y}, 1} \\
\mathrm{H}_{\mathrm{z}, 1}=\left(\mathrm{ck} / \omega \mu_{1}\right) \mathrm{E}_{\mathrm{y}, 1} \\
E_{y, 2}=\left[E_{2}^{(1)} \exp \left(p_{2} z\right)+E_{2}^{(2)} \exp \left(-p_{2} z\right)\right] \exp [i(k x-\omega t)] \quad 0<\mathrm{z}<\mathrm{d} \\
\mathrm{H}_{\mathrm{x}, 2}=(\mathrm{ic} / \omega)\left(\partial E_{y, 2} / \partial z\right)
\end{gathered}
$$


International Journal of Recent advances in Physics (IJRAP) Vol.4, No.4, November 2015

$$
\begin{gathered}
\mathrm{H}_{\mathrm{z}, 2}=(\mathrm{ck} / \omega) \mathrm{E}_{\mathrm{y}, 2} \\
\mathrm{E}_{\mathrm{y}, 3}=\mathrm{E}_{3} \exp \left[\mathrm{i}(\mathrm{kx}-\omega \mathrm{t})+\mathrm{p}_{3} \mathrm{z}\right] \quad \mathrm{z}<0 \\
H_{x, 3}=\left(i c p_{3} / \omega\right) E_{y, 3} \\
\mathrm{H}_{\mathrm{z}, 3}=(\mathrm{ck} / \omega) \mathrm{E}_{\mathrm{y}, 3}
\end{gathered}
$$

$\mathrm{H}_{\mathrm{y}}=\mathrm{E}_{\mathrm{x}}=\mathrm{E}_{\mathrm{z}}=0$

By taking the boundary conditions at the interface $\mathrm{z}=\mathrm{d}$, and the presence of a surface current at the interface $\mathrm{z}=0$ leads to discontinuity of the magnetic field tangential components giving the following equations:

$$
\begin{aligned}
& H_{x, 2}^{(s)}-H_{x, 3}^{(s)}=\frac{4 \pi}{c}\left(\sigma_{x x} E_{y}^{(s)}-\sigma_{x y} E_{x}^{(s)}\right. \\
& H_{y, 2}^{(s)}-H_{y, 3}^{(s)}=\frac{4 \pi}{c}\left(\sigma_{x x} E_{x}^{(s)}-\sigma_{x y} E_{y}^{(s)}\right.
\end{aligned}
$$

where, $\sigma_{i j}(\omega)$ are the 2DES conductivity tensor components, and the index s indicates the values of the electric and magnetic fields at the interface $\mathrm{z}=0$. We assume that the spatial dispersion of the conductivity tensor can be neglected, i.e. $k l \prec \prec 1$, where $l=(c \bar{h} / e B)^{1 / 2}$ is the magnetic length $[5,6]$ :

$$
\begin{gathered}
\sigma_{x x}=\frac{2 e^{2}}{h} \frac{N \gamma}{1+\gamma^{2}} \\
\sigma_{x y}=\frac{2 e^{2}}{h} \frac{N}{1+\gamma^{2}}
\end{gathered}
$$

where $\gamma=(\nu-i \omega) / \Omega$, and $\Omega=e B / m c$ is the cyclotron frequency; $v$ is the momentum relaxation frequency of the electrons; and $N=\pi l^{2} n$ is the Landau-level filling factor and has an integer values $(\mathrm{N}=1,2, \ldots)$ which equal to the numbers of filled Landau levels lying below the Fermi level.

By using the boundary conditions at the interfaces $\mathrm{z}=0$ and $\mathrm{z}=\mathrm{d}$, the dispersion equation of the non-irradiative SPs can be derived:

Where

$$
A_{1} A_{2}+\left(4 \pi \sigma_{x y} / c\right)^{2} p_{2} p_{3} B_{1} B_{2}=0
$$




$$
\begin{gathered}
A_{1}=\left(p_{3} \varepsilon_{2}-p_{2} \varepsilon_{3}-\left(i 4 \pi p_{2} p_{3} \sigma_{x x} / \omega\right)\right)\left(p_{2} \varepsilon_{1}-p_{1} \varepsilon_{2}\right)+ \\
\exp \left(2 p_{2} d\right)\left(p_{3} \varepsilon_{2}+p_{2} \varepsilon_{3}+\left(i 4 \pi p_{2} p_{3} \sigma_{x x} / \omega\right)\right)\left(p_{2} \varepsilon_{1}+p_{1} \varepsilon_{2}\right) \\
A_{2}=\left(p_{2}-p_{3}+\left(i 4 \pi \omega \sigma_{x x} / c^{2}\right)\right)\left(\mu_{1} p_{2}-p_{1}\right)- \\
\exp \left(2 p_{2} d\right)\left(p_{2}+p_{3}-\left(i 4 \pi \omega \sigma_{x x} / c^{2}\right)\right)\left(\mu_{1} p_{2}+p_{1}\right) \\
B_{1}=\mu_{1} p_{2}-p_{1}+\exp \left(2 p_{2} d\right)\left(\mu_{1} p_{2}+p_{1}\right) \\
B_{2}=p_{2} \varepsilon_{1}-p_{1} \varepsilon_{2}+\exp \left(2 p_{2} d\right)\left(p_{1 \varepsilon_{2}}+p_{2} \varepsilon_{1}\right)
\end{gathered}
$$

In our study, we take the dimensionless frequency $\xi=\omega / \Omega$, the dimensionless wave vector $\zeta=\mathrm{ck}$ $/ \Omega$, and the dimensionless thickness $\delta=\mathrm{d} \Omega / \mathrm{c}$. It was published [30] that when considering the limiting cases in which the 2DES is located between the two-infinite media, i.e. $\delta=\infty$ and $\delta=0$. The dispersion curves $\xi(\zeta)$ for Surface Polaritons (SP) were shown for two cases: layer 1 is a dielectric (a vacuum or an air) where $\varepsilon_{1}=1$ while $\varepsilon_{2}=12.0$ and $\varepsilon_{3}=12.9$. The numbers 1 and 5 indicate the values of $\mathrm{N}$ considered in our analysis and the finite value of the dimensionless thickness $\delta=0.7$. It was found [10] that the dispersion curves for the case where $\varepsilon_{2}=\varepsilon_{3}=12, \delta$ $=\infty$, the point at which dispersion curves intersect, $\zeta \infty=\mathrm{ck} \infty / \Omega$, is located to the right of the one obtained when $\delta=0$. In the case where $\delta=\infty$, the larger value of the dielectric constant of GaAs substrate (in comparison with the dielectric constant of AlxGa1-xAs) leads to decrease of the phase and the group velocities of the SPs in the vicinity of the CR. If $\delta$ decrease, the phase and the group velocities of the SPs increase and they reach maximum values at $\delta=0$. The difference between the dielectric constants of two media, 2 and 3, leads to an interesting new results, i.e., all dispersion curves start at the light line for the GaAs medium.

\section{RESULTS AND DISCUSSIONS}

In the calculation, several sets of $\varepsilon_{1}$ and $\mu_{1}$ of the LH material are considered with their products kept the same i.e., $\varepsilon_{1} \mu_{1}=4$ as shown in table 1[30]. In figure 2, the dispersion curves for the SP are presented where the dimensionless frequency is plotted against the dimensionless wave vector. In figure 2 (a) the upper layer of the structure is considered to a vacuum or an air (dielectric), i.e. $\varepsilon_{1}=1$ as published before [31] while in figure 2 (b) a Left Handed (LH) material is introduced as an upper layer where $\varepsilon_{1}=-2, \mu_{1}=-2$, while $\varepsilon_{2}=12.0$ and $\varepsilon_{3}=12.5$. This has been conducted to compare between our current structure presented in this work and similar structure published before. The only difference between the two structures is the replacement of the upper layer to be a LH material rather than a dielectric in previous work. Figure 2 (a) and (b) had been computed for the definite values of the dimensionless thickness $\delta=0.65$ and for the values of the Landen level filling $\mathrm{N}=1$ ( solid) line, $\mathrm{N}=5$ (dotted line) and $\mathrm{N}=10$ (dashed line). It can be seen from figure 2 (a) that the intersection point in all curves is $(5,1)$ and the change of the frequency versus wave vector is more pronounced with increasing of $\mathrm{N}$; there is very slight difference of the values of the frequency with the change of the wave vector at $\mathrm{N}=5$. In Figure 2 (b) it is obvious the effect of introducing the LH upper layer. At wave number values less than 5 it can be seen the rapid increase of the operating frequency versus the increase of wave number. At wave number of 5 the frequency equals 1.22. This is the benefit of the LH layer. At wave number is more than 5 the behavior of the frequency versus wave number is very similar to the traditional structure where the upper layer is dielectric. 
Different situation is there in figure 3 where the dispersion curves for the SPs is presented as $\varepsilon_{2}$ and $\varepsilon_{3}$ were kept equals and have the values of 12.0 , for two values of the dimensionless thickness: $\delta=0.1$ for the dashed line and $\delta=1$ for the solid line and for unique value of $\mathrm{N}=1$. It can be noticed from the figure that the frequency has a cut off at wave number $=4.9$ for $\delta=1$ and a wave vector $=5.85$ for $\delta=0.1$, similar to the above case as shown in figure 2 . In both cases the frequency starts to appear again directly after the cut off value of the wavenumber but with very lower value nearly equals 1 . In the dashed line and with decreasing the thickness of factor of ten, it is shown that the rapid increase of frequency versus wave vector continues up to wave vector of 5.85. This shows the importance of the decrease of the thickness of LH material upper layer in the performance of the structure. Moreover it is noticed that a higher value of frequency of 1.4 can be obtained at a wave vector $=5.85$.
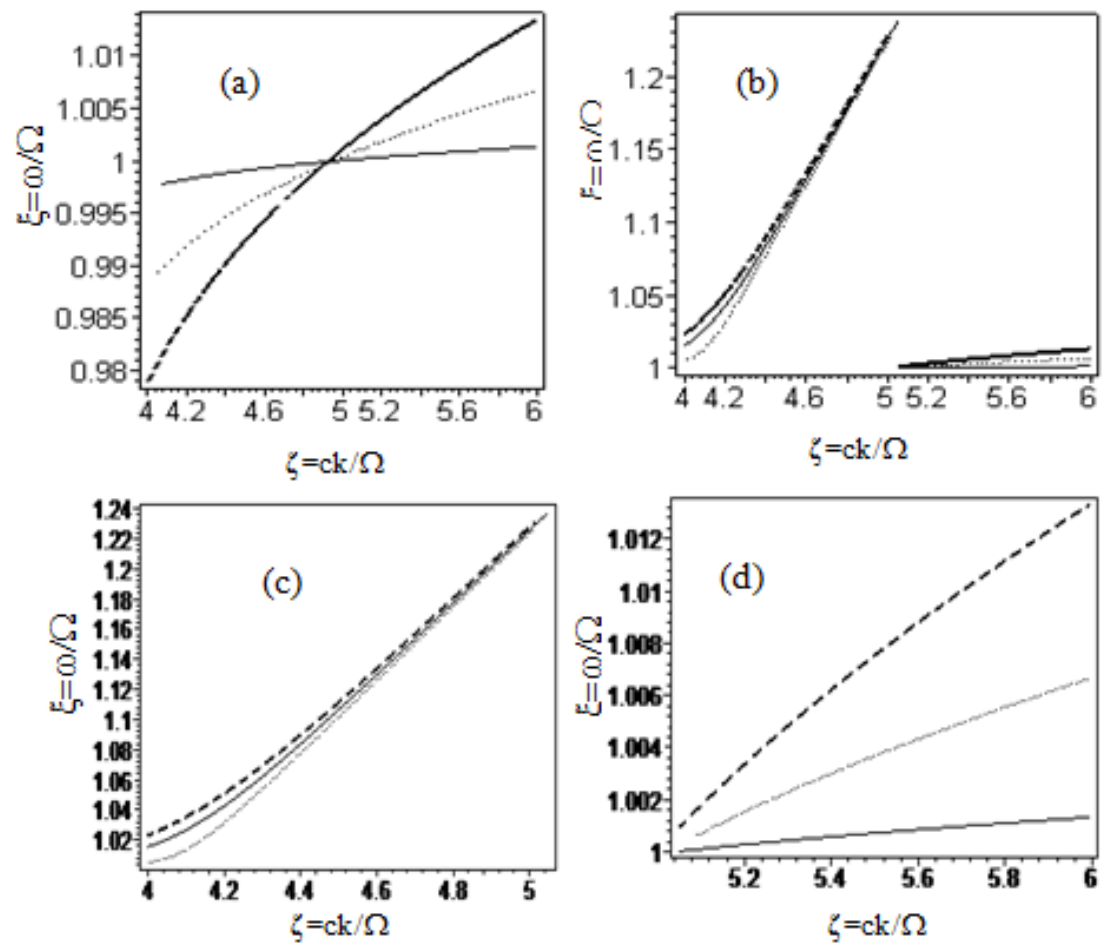

Fig 2 The dispersion curves for the SPs in the case in (a) which 21 $=1$ and (b) $\left.\varepsilon_{1}=-2, \mu\right]=-2, \varepsilon_{2}=12.0, \varepsilon_{3}=12.5$, and for the finite values of the dimensionless thickness $\delta=0.65$ and for the values of $N=1$ (solid line), $\mathrm{N}=5$ (dotted line), and $\mathrm{N}=10$ (dashed line). (c) and (d) is (b) but split in two figures and magnified to highlight the curves.

Table 1

Combination of $\varepsilon_{1}$ and $\mu_{1}$ of LH material in the present study

\begin{tabular}{|l|l|}
\hline$\varepsilon_{1}$ & $\mu_{1}$ \\
\hline-8 & -0.5 \\
\hline-5 & -0.8 \\
\hline-0.5 & -8 \\
\hline-2 & -2 \\
\hline-1 & -4 \\
\hline-0.8 & -5 \\
\hline
\end{tabular}


International Journal of Recent advances in Physics (IJRAP) Vol.4, No.4, November 2015
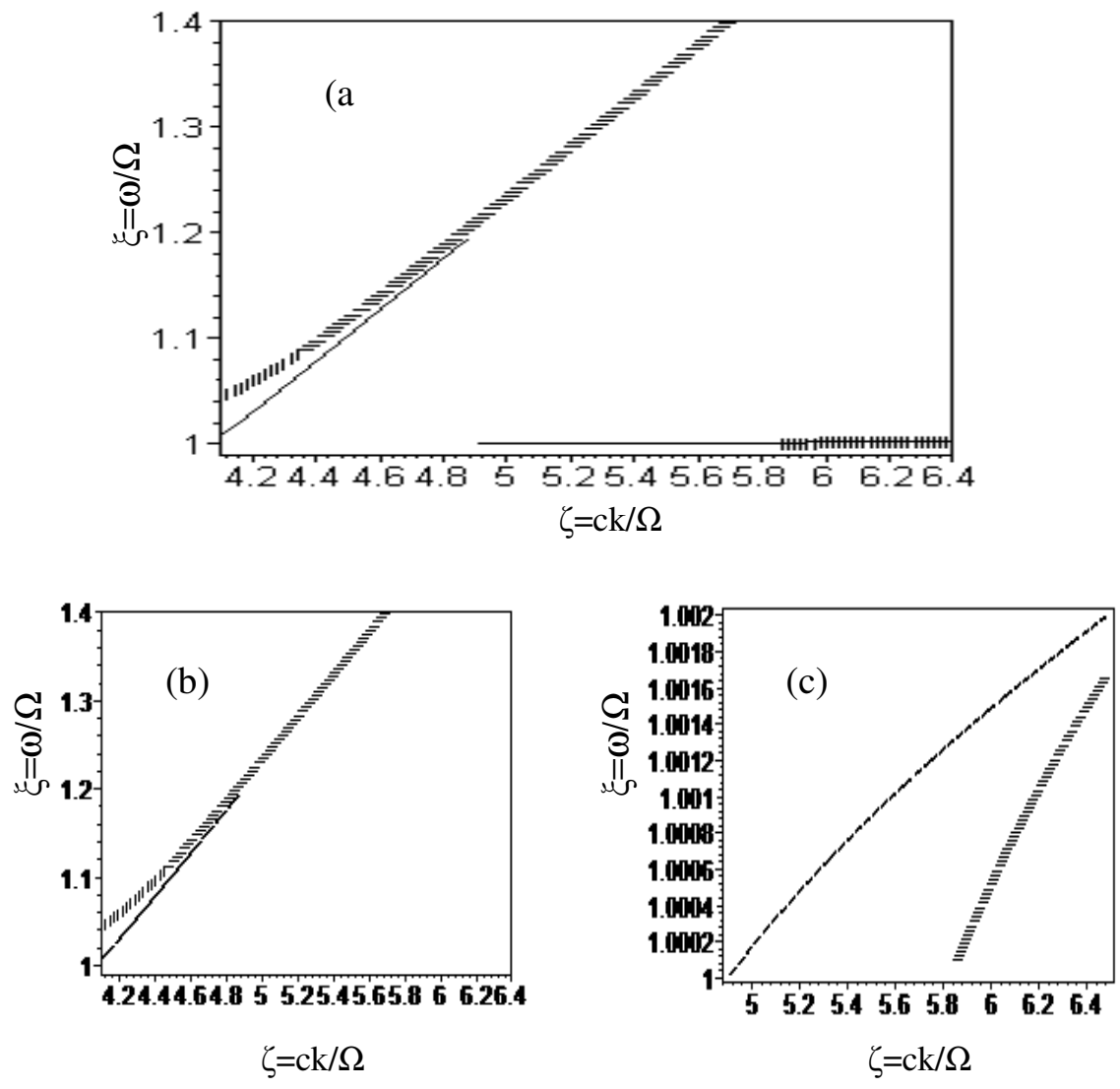

Fig. 3. The dispersion curves for the SPs in the case in which $\varepsilon 1=-2, \mu 1=$ $-2, \varepsilon 2=12.0, \varepsilon 3=12.0$, and for the finite values of the dimensionless thickness $\delta=0.1$ (dashed line) and $\delta=1$ (solid line) and for $\mathrm{N}=1$. (b) and (c) is (a) but split in two figures and magnified to highlight the curves. 

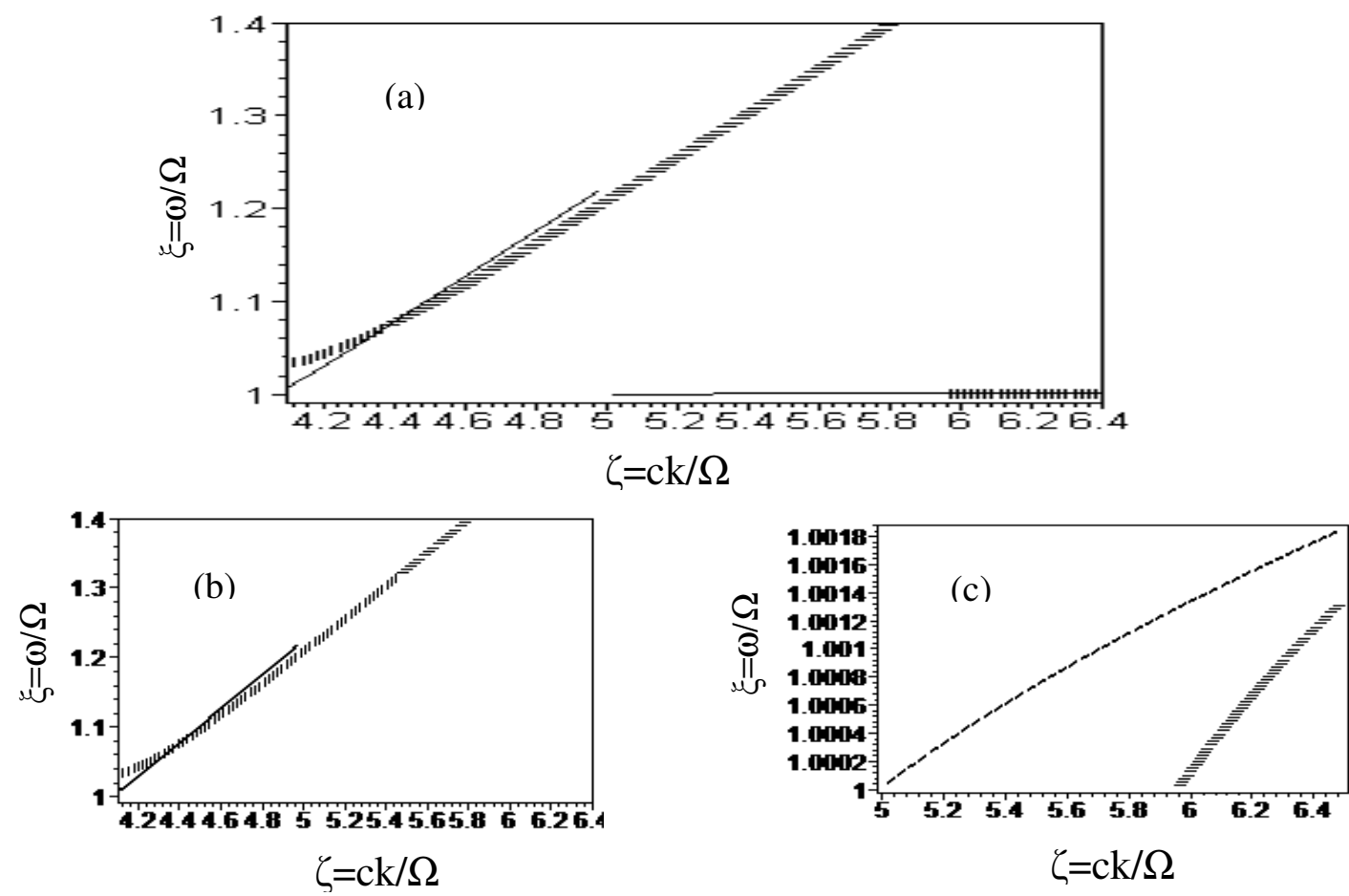

Fig. 4. The dispersion curves for the SPs in the case in which $\varepsilon_{1}=-2, \mu_{1}=-2, \varepsilon_{2}=12.0, \varepsilon_{3}$ $=12.9$, and for the finite values of the dimensionless thickness $\delta=0.1$ (dashed line) and $\delta$ $=1$ (solid line) and for $\mathrm{N}=1$. (b) and (c) is (a) but split in two figures and magnified to highlight the curves.

In figure 4 the dispersion curves for the SPs is presented where $\varepsilon_{2}=12.0$ and $\varepsilon_{3}=12.9$, i.e. they are not equal as in figure 3, while for the same two values of the dimensionless thickness used in figure 3: $\delta=0.1$ for the dashed line and $\delta=1$ for the solid line and also for unique value of $\mathrm{N}=1$ used in figure 3 .

It can be observed from the figure 4 that the frequency has a cut off at wave vector $=5$ for $\delta=1$ and a wave vector $=6$ for $\delta=0.1$. It is clear that there is slightly increase of the wave vector values of the cut off when $\varepsilon_{3}$ is slightly increased from $\varepsilon_{2}=12.0$ (figure 3 ) to $\varepsilon_{3}=12.9$ (figure 4). It is also noticed that there is slightly increase of the frequency values of the cut off from 1.18 (at $\left.\varepsilon_{2}=12.0\right)$ to $1.22\left(\varepsilon_{3}=12.9\right)$ in the solid line $(\delta=1)$.

Different situation is considered in figure 5 where the curves are plotted in different manner. In the previous figures 2,3,4: $\varepsilon_{1}$ and $\mu_{1}$ are fixed and both kept equal -2 , and the variation is in the other parameters, i.e., $\varepsilon_{2}, \varepsilon_{3}$ and the dimensionless thickness $\delta$. Here in figure 5: $\varepsilon_{2}$ and $\varepsilon_{3}$ were fixed and kept equal 12 and the dimensionless thickness $\delta$ is also fixed while $\varepsilon_{1}$ and $\mu_{\mathrm{LH}}$ were varied and have different values. As can be seen in figure 5 (a), four curves are plotted where all the curves have the same behavior and looks parallel. The frequency is higher and more pronounced ( Maximum frequency $=1.4$ ) in curve 4 where $\varepsilon_{1}$ has the highest value $=-0.5$ while $\mu_{1}$ has the lowest value $=-8$. The lowest frequency (Maximum frequency $=1.18$ ) is obtained in curve 1 where $\varepsilon_{1}=-2$ and $\mu_{1}=-2$. This data show the sensitivity and importance influence of the left- handed layer's parameters upon the value of the obtained frequency which should be considered in the proposed structure. It is also noticed in figure 5(b) that despite the variation in 
the lift- handed layer's parameters the cut off in the four curves occur at the same wave vector value.
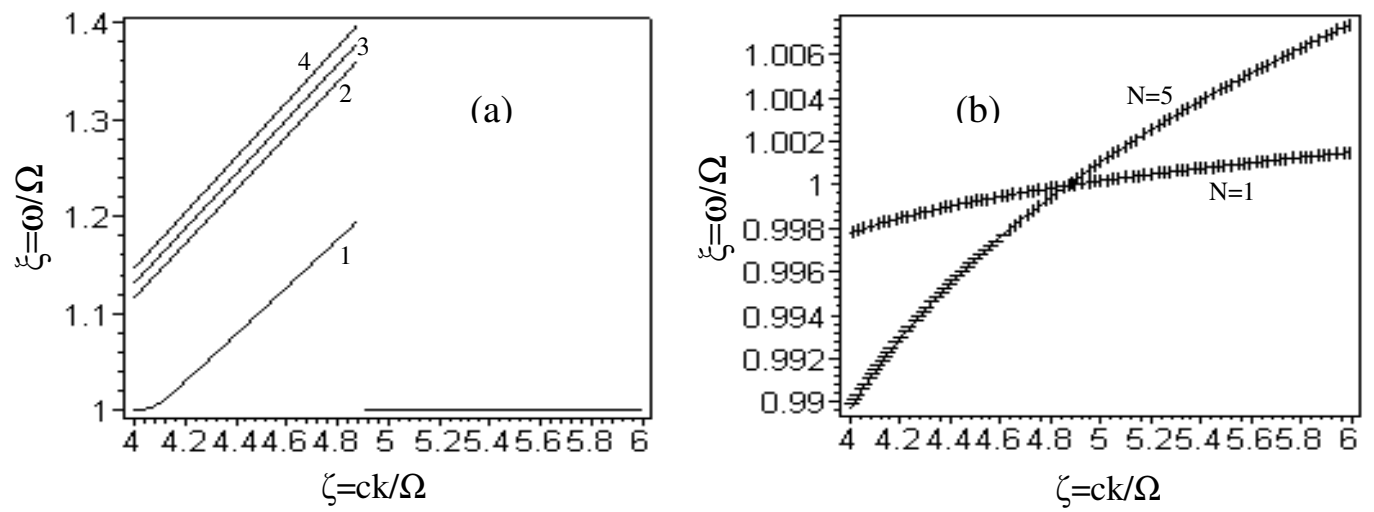

Fig. 5 The dispersion curves for the SPs in the case in which $\varepsilon_{2}=12.0, \varepsilon_{3}=12$, and for the finite values of the dimensionless thickness $\delta=1$ in the case (a) $1\left(\varepsilon_{1}=-2, \mu_{1}=-2\right), 2\left(\varepsilon_{1}=-1\right.$, $\left.\mu_{1}=-4\right), 3\left(\varepsilon_{1}=-0.8, \mu_{1}=-5\right), 4\left(\varepsilon_{1}=-0.5, \mu_{1}=-8\right),(b)$ in the case: $1\left(\varepsilon_{1}=-8, \mu_{1}=-0.5\right), 2\left(\varepsilon_{1}=-\right.$ $\left.5, \mu_{1}=-0.8\right)$,

In figure 5 (b) very low values of $\varepsilon_{1}$ are chosen: $\varepsilon_{1}=-8$ (case 1 ) and $\varepsilon_{1}=-5$ (case 2) whereas high values of $\mu$ were considered: $\mu_{1}=-0.5$ (case 1) and $\mu_{1}=-0.8$ (case 2). In this case very nice results have been noticed; two intersected curves were seen using $(\mathrm{N}=1$ and $\mathrm{N}=2)$. The features of these curves which obtained with left-handed material upper layer of the structure are similar to the results ( which presented in figure 2(a) )obtained using dielectric (vacuum) upper layer.

\section{CONCLUSIONS}

We have calculated the spectrum of the SPs in the $\mathrm{GaAs} / \mathrm{Al}_{\mathrm{x}} \mathrm{Ga}_{1-\mathrm{x}} \mathrm{As} / \mathrm{LH}$ heterojunction in a high magnetic field, i.e. for a case in which the effects of quantization of the conductivity tensor of a 2DES are crucial. It is shown that all of the dispersion characteristics of the SPs under the conditions of the integer quantum Hall effect are quantized. In the vicinity of the cyclotron resonance, the phase and group velocities of the SPs are decreasing significantly.

The values of the dimensionless frequency versus dimensionless wave vector of the SPs can strongly effected by using the LH upper layer in the proposed structure. These values are also influenced with increasing thickness $\mathrm{d}$ of the $\mathrm{Al}_{\mathrm{x}} \mathrm{Ga}_{1-\mathrm{x}}$ As layer, and with the variation of the difference between the dielectric constants of GaAs and $\mathrm{Al}_{\mathrm{x}} \mathrm{Ga}_{1-\mathrm{x}}$ As. This fact can be used in various applications in microelectronics and in making contactless measurements of the parameters of $\mathrm{GaAs} / \mathrm{Al}_{\mathrm{x}} \mathrm{Ga}_{1-\mathrm{x}} \mathrm{As} / \mathrm{LH}$ heterojunction. Investigations of the SPs using inelastic light scattering are of important application for measuring the Landau-level filling factor $\mathrm{N}$ dependence by using dispersion data with no direct contact with the $\mathrm{GaAs} / \mathrm{Al}_{\mathrm{x}} \mathrm{Ga}_{1-\mathrm{x}}$ As/LH heterojunction.

\section{ACKNOWLEDGEMENTS}

One of the Authors (M.M.S) gratefully acknowledge financial grant from the Alexander von Humboldt Foundation and the hospitality of Prof. D.Schadaat, at the Clausthal University of Technology, Germany 
International Journal of Recent advances in Physics (IJRAP) Vol.4, No.4, November 2015

\section{REFERENCES}

[1] V. M. Agranovich and D. L. Mills (Ed) (1982), " Surface Polaritons. Electromagnetic Waves at Surfaces and Interfaces" (Amsterdam: North Holand).

[2] A. D. Boardman (Ed) (1982), "Electromagnetic Surface Modes" (New York: Wiley).

[3] T. Ando, B. Fowler and F. Stem, " Electronic properties of two-dimensional systems " Rev. Mod. Phys. Lett. 54 (1982) 437.

[4] Yu. A. Kosevich, A. M. Kosevich and J. C. Granada, (1988) " Magnetoplasma oscillations of a twodimensional electron layer in a bounded system"Phys. Lett. 127A 52.

[5] I. E. Aronove and N. N. Beletskii, (1996) " Surface polaritons in a GaAs/AlGaAs heterojunction in a high magnetic field" J. Phys.: Condens. Matter 84919.

[6] I. E. Aronove, N. N. Beletskii, G.P. Berman and A. R. Bishop (1997) "Collective electromagnetic excitations in a double-layer two-dimensional electron system in a high magnetic field" Phys. Rev. B 5610392.

[7] N. C. Constantinou and M. G. Cottam, (1986) " Surface polaritons in a GaAs/AlGaAs heterojunction in a high magnetic field J. Phys.: Condens. Matter 19739.

[8] R. F. Wallis and J. J. Quinn, (1988) " Surface magnetoplasmon polaritons in truncated semiconductor superlattices", Phys. Rev. B 384205

[9] Yu Liming and Wang Qi,(1999)." Stability Of Magnetostatic Surface Waves In A SemiconductorFerrite-Left-Handed Material Waveguide Structure"J. of Shanghai Univer. 31

[10] V. G. Veselago, (1964) " The electrodynamics of substances with simultaneously negative values of $\varepsilon$ and $\mu$ "Usp. Fiz. Nauk 92517.

[11] J. B. Pendry et al, (1999) " Magnetism from conductors and enhanced nonlinear phenomena" Microwave Theory Tech. 47, 2075.

[12] J. B. Pendry, A. J. Holden, W. J. Stewart and I. Youngs, (1996) " Extremely Low Frequency Plasmons in Metallic Mesostructures"Phys. Rev. Lett. 76, 4773

[13] R. M. Walser, A. P. Valanju, P. M. Valanju, Phys. (2001) " Comment on "extremely low frequency plasmons in metallic mesostructures"."Rev. Lett. 87119701.

[14] D. R. Smith, W. J. Padilla, D. C. Vier, S. C. Nemat-Nasser and S. Schultz, (2000) " Composite Medium with Simultaneously Negative Permeability and Permittivity" Phys. Rev. Lett. 844184.

[15] J. B. Pendry, (2000) " Negative Refraction Makes a Perfect Lens"Phys. Rev. Lett. 85, 3966.

[16] D. R. Smith, D. Schurig, and J. B. Pendry, (2002) " Negative refraction of modulated electromagnetic waves"Appl. Phys. Lett. 81, 2713.

[17] J. Pacheco, T. M. Grzegorczyk, B. I. Wu, Y. Zhang and J. A. Kong, (2002) " Power Propagation in Homogeneous Isotropic Frequency-Dispersive Left-Handed Media" Phys. Rev. Lett. 89257401.

[18] N. Garcia and M. Nieto-Vesperinas, (2002)" Left-Handed Materials Do Not Make a Perfect Lens"Phys. Rev. Lett. 88, 207403. (2002)

[19] P. M. Valanju, R. M. Walser, and A. P. Valanju," Wave Refraction in Negative-Index Media: Always Positive and Very Inhomogeneous" Phys. Rev. Lett. 88187401.

[20] R. Rupin, (2000) " Surface polaritons of a left-handed medium"Phys. Lett. A 22761.

[21] R. Rupin, (2000) " Extinction properties of a sphere with negative permittivity and permeability"Solid State Commun. 116411.

[22] V. Kuzmail and A. A. Maradudin, (2002) " Scattering properties of a cylinder fabricated from a lefthanded material"Phys. Rev. B 66045116.

[23] R. W. Ziolkowski and E. Heyman, (2001) " Wave propagation in media having negative permittivity and permeability"Phys. Rev. E 64056625.

[24] F. J. Rachford, D. L. Smith, P. F. Loschialpo and D. W. Forester, (2202) " Calculations and measurements of wire and/or split-ring negative index media" Phys. Rev. E 66036613.

[25] M. S. Hamada, A. H. El-Astal and M. M. Shabat, (2007) " Characteristic of Surface Waves in Nonlinear Left Handed Photosensitive Semiconductor Waveguide Structure " Int. J. Modern Physics B21 1817.

[26] M. Hamada, M. M. Shabat and D. Jager, (2003) " Nonlinear TM surface waves in a Left-Handed material structure " Proc. Of SPIE 5445184.

[27] M. S. Hamada, A. H. El-Astal and M. M. Shabat, (2007) " Non-Linear Surface Waves At a Single Interface of Semimagnetic Semiconductor - Left Handed Materials (LHM), "Int. J. Microwave and Optical Tech. 2(2) 112. 
International Journal of Recent advances in Physics (IJRAP) Vol.4, No.4, November 2015

[28] H. M. Mousa and M. M. Shabat, (2007), " Non linear TE Surface in a Left-Handed Material and Superlattices Wave-Guide structures,"Int. J. Modern Physics B 21(6), 895.

[29] M. Nakayama, (1974)" Theory of Surface Waves Coupled to Surface Carriers" J. Phys. Soc. Japan 36 393.

[30] K. Y. Kim, Y. K. Cho, and H-S Tae, (2006)," Guided Mode Propagations of Grounded DoublePositive and Double-Negative Metamaterial Slabs with Arbitrary Material Indexes" J. of the Korean Phys. Society, 49(2), 577

[31] N. N. Beletskii, G. P. Bermant, A. R. Bishop and S. A. Borisenko(1998)"Surface polarions in a GaAs/AlGaAs heterjuntion in a high magnetic field", J. Phys. : Condens. Matter 105781. 